\title{
Hartmut Elsenhans
}

Ökonomistische oder politökonomische Analyse der ölkrise Anmerkungen zu den Thesen won M. Massarrat

(in Proklla 23)

Die Ölkrise als Ausdruck des Entstehens einer Kapitalistenklasse in den Ölexportländern: mit dieser Aussage will Massarat die von Bassam Tibi und meinen Arbeiten linksbürgerlich unterwanderte Linke in Deutschland wieder auf einen korrekten ,politökonomischen“" Kurs bringen, dessen Praxisbezug er im Gegensatz zu dem reformistischen, dem Kapital verhafteten Ansatz meiner Arbeit darin sieht, den Arbeitern in den Industrieländern zu erklären, daß sie von dieser neuen Kapitalfraktion in den Ölländern ausgebeutet werden (S. 17) (1). Aus der Unterstützung der Perspektiven, die die ölländer für alle Rohstoffländer der Dritten Welt eröffnet haben, indem sie den Weg zur Vergrößerung der Akkumulationsfonds gezeigt haben und zumindest in einzelnen Fällen die Finanzierungsmit tel für neue Umverteilungsmaßnahmen bereitstellen, soll offenbar die westdeutsche Linke herausgehalten werden, und dazu müssen natürlich die Theoretiker einer solchen praktischen Solidarität zwischen den Arbeiterklassen in den Metropolen und den Massen in der Dritten Welt widerlegt werden.

Diese Widerlegung versucht Massarat - abgesehen von einer Vielzahl von Fehlinterpretationen meiner Arbeiten, auf die hier nicht eingegangen werden soll - mit Hilfe von drei Argumentationen zu leisten:

- Auf einer empirischen Ebene versucht Massarat meine These zu widerlegen, daß sich durch die Auslagerung der Energieproduktion in die Dritte Welt seit Mitte der 50er Jahre die Kosten der Energie für die Vereinigten Staaten im Verhältnis zu den Kosten der (importierten) Energie in den westeuropäischen Staaten und Japan erhöht haben. Zweck der Widerlegung dieser These ist, den Interessengegensatz zwischen Westeuropa und den USA zu leugnen und damit dem Argument, daß die Ölländer aufgrund der besonderen Interessenlage Japans und Westeuropas in neuen Formen der Kooperation zwischen Westeuropa und Japan die Überwindung von Unterentwicklung beschleunigen können, den Boden zu entziehen.

- Auf einer theoretischen Ebene versucht Massarat mir nachzuweisen, daß die Entwickiungstendenzen der Gesellschaften in der Dritten Welt von mir nicht analysiert seien und deshalb der gesellschaftliche Charakter der Preissteigerungen für o̊l nicht erkannt werde - nämlich deren notwendig kapitalistiscer Charakter. Offensichtlich wird hier die Zielsetzung, alle Reformversuche im Bereich der Weltwirtschaftsbeziehungen als kapitalistische zu diskreditieren, weil ohne Umsturz des Kapitalismus auf weltweiter Ebene strukturverändernde Reformen in der Dritten Welt als weiterhin unmöglich hingestellt werden müssen (S. 33).

- Auf einer wissenschaftstheoretischen Ebene versucht Massarat meinen Arbeiten zur Erdölkrise eine politologische Beschränkung und empirische Detailhuberei (S. 25, S. 5) nachzuweisen, um für sich die theoretische Klarheit des Alles-Erklären-Könnens zu beanspruchen.

Alle drei Argumentationen Massarats sind allerdings falsch.

\section{War das Energieniveau in den USA vor der Energiekrise höher als in Westeuropa?}

Massarat glaubt in der Frage des Energiepreisniveaus, dem einzigen Abschnitt, bei dem er Empirie heranzieht, mir grobe Fehler bei der Aufarbeitung des empirischen Materials nachweisen zu können. „, Der Fehler von $\mathrm{E}$, scheint in einer Verwechslung von verschiedenen Freisebenen zu. liegen $^{6}$, weil ich den O̊lmarktpreis von $3,30 \$ / 1$ mit dem Öllistenpreis für OPEC-Öl verglichen

1 Die Seitenangaben im Text beziehen sich auf Massarat, M.: Linksbürgerliche Analysen der ,Energiekrise". Versuch einer Wissenschafts- und Ideologikritik, in: Prokla 23, S. 17 -54 . 
hätte (S. 43 f.). Massarat hat ganz recht mit der Behauptung, beides seien verschiedene Preise, aber ganz unrecht mit der Behauptung, der Preis von $3,30 \$ / \mathrm{b}$ oder ca $24 \$ / \mathrm{t}$ in den USA müsse mit dem von ihm errechneten ,Ölmarktpreis“ in Westeuropa von $57-58 \$ / t$ vor der Ölkrise verglichen werden (S. 44).

Massarats „Ölmarktpreis“ von $3,30 \$ / \mathrm{b}$ in den USA ist tatsächlich der Listenpreis für Öl in den USA, der $a b$ Feld bezahlt werden muß. Für die amerikanischen Energieverbraucher erhöht sich dieser Preis um die Kosten von Transport und, sofern es sich nicht um Rohölkäufer handelt, Verarbeitung. Der Öllistenpreis für Nahostöl ist dagegen ein Steuerreferenzpreis, d. h. ein fiktiver Preis, auf dessen Grundlage die Abgaben (Royalties und Steuern) der Konzerne an die Förderländer berechnet wurden. Der Produktionspreis für die Konzerne nach Steuer- und Royaltyleistung war keineswegs der Listenpreis, sondern die Taxpaid costs, d. h. 1973 etwa $60 \%$ des Listenpreises, als etwa $1,75 \mathrm{\$} / \mathrm{b}(2)$. Um die Kosten von amerikanischen und Nahostöl in den Verbrauchszentren in Westeuropa, Japan und den USA zu vergleichen, muß pro Faß Nahostöl noch ca. 1 \$ für den Transport zu den Tax-paid costs hinzugerechnet werden. Dann erhalten wir den Preis für Nahostöl in Westeuropa vor Zollerhebung, der dann wohl mit dem amerikanischen Rohölpreis von $\$ 3,30$ pro Faß verglichen werden kann und um $50-60$ cts. niedriger war als der amerikanische ölpreis $a b$ Feld. Das bedeutet, daß die Konzerne Rohöl in den Zentren der Verarbeitung und des Verbrauchs Westeuropas etwa um $20 \%$ billiger liefern konnten als in den USA $a b$ Ölfeld.

Diesen Befund versucht Massarat zu umgehen, indem er den Erlös für die verarbeiteten Produkte aus einer Tonne öl in Westeuropa mit dem Marktpreis für Rohöl in den USA vergleicht. Massarat behauptet einen Preis von $55-57 \$ / t$ Öl; d. h. von 155 DM pro t (S. 44). Da eine Tonne Öl etwa $1150 \mathrm{l}$ ergibt, beträgt der durchschnittliche Preis pro Liter 15 Pf. Da die Verbraucher z. B. in der Bundesrepublik vor der Energiekrise für 22,5\% der Ölprodukte, nämlich schweres Heizöl, nur $9-10$ Pf pro Liter, und weitere 38,3\% der Produkte 15-16 Pf pro Liter bezahlten, (3) kann Massarats Marktpreis nur die Summe der Erlöse für alle Ölprodukte sein. Den Rohölpreis ab Feld in den USA und den Erlös für die Produkte aus einer verarbeiteten Tonne Rohöl zu vergleichen, heißt Äpfelpreise mit Apfelkuchenpreisen zu vergleichen.

Aber Massarat hat einen weiteren empirischen Befund, nämlich die Angaben über die Preise von Erdölprodukten im Statistischen Bulletin der OPEC von 1974 (S. 43). Diese Zahlen sind von uns nicht verwendet worden, weil sie außerordentlich unsystematisch (keine Durchschnittspreise) erhoben sind und nur Teilmärkte erfassen. Im Gegensatz zu Massarats Behauptung umfassen sie die für das Energiepreisniveau wichtigen Heizöle nicht, sondern nur Bunkeröl, was Massarat als Heizöl übersetzt. Bunkeröle sind aber nur die Öle, die an Schiffe zum „Einbunkern“ geliefert werden.

Massarats Statistik widerlegt ihn aber noch selbst: Sie zeigt zwar, daß Benzine und Bunkeröle in den USA im Einzelhandel billiger waren als in Westeuropa (was weiter unten für Heizöle widerlegt wird), sie zeigt aber auch, daß diese niedrigeren Kosten ausschließlich Folge der in den USA niedrigeren Steuerbelastung waren. So kostet nach Abzug von Steuern Benzin in den USA $27,6 \mathrm{cts}$./Gallon (New York) bzw. 26,6 cts./Gallon (San Francisco). In Italien beträgt dagegen dieser Preis 22,7 cts (wobei $6 \%$ Zoll noch enthalten sind) und in Frankreich nur 25,5 cts. Da Rohbenzine die Grundst offe der Petrochemie sind, nicht der Mineralölsteuer unterliegen werden, liefert Massarats Statistik den Nachweis dafür, daß die Petrochemie in Europa niedrigere Rohstoffkosten hat te als in den USA. Genauso zeigen die Preise für Bunkeröle, daß nach Abzug von Steuern die Peise in Italien, Frankreich und Großbritannien niedriger als in San Francisco sind und nur z. T. höher als in New York liegen, wo nämlich Ölprodukte aus Nahostöl umgesetzt werden.

Massarats Statistik scheint also den Schluß nahezulegen, daß zwar die Kosten für Erdölprodukte für die westeuropäischen Volkswirtschaften, nicht aber für die Verbraucher niedriger

2 Vgl. dazu ausführlich Elsenhans, H.: Die Kostensteigerungen für Erdöl im Juni 1973 bis Januar 1974. Schriftenreihe des Fachbereichs Politische Wissenschaft, Nr. 5, Freie Universität Berlin. Berlin 1974, v.a. S. $15-24$. Ebenda, S. 43. 
waren, so daß sich die Regierungen in der EG anders als die Regierung der USA einen Teil der Differentialrente aneigneten, nicht aber die energieintensiven Branchen der westeuropäischen Industrie gegenüber der amerikanischen konkurrenzfähiger wurden,

Aber selbst diese Schlußfolgerung ist falsch, wenn repräsentativeres Datenmaterial herangezogen wird: Das Statistische Bundesamt erhebt eine Reihe von Preisen für Endprodukte. Deren Preisvergleich ist dadurch erschwert, weil die Preise entweder ab Raffinierie oder frei Huas angegeben werden. Vergleichbar sind Preise, die einen einheitlichen Anteil an Dienstleistungen enthalten, wobei die breitest gestreuten Angaben sich auf die Lieferung ab Raffinerie auf Tankwagen beziehen, für die Angaben für schweres Heizöl (Bundesrepublik, Niederlande, Italien) und leichtes Heiöl (New York, Italien-vorliegen. Diese Preise, denen wir die Preise für $100 \mathrm{~kg} \mathrm{Koh-}$ le in den USA und der Bundesrepublik hinzufugen, zeigen ein eindeutig niedrigeres Preisniveau für Energie in Westeuropa (4).

Sept. 1972 Dez.1972 März 1973 Aug. 1973

DM pro 1001

Heizö1 leicht

Italien

New York

Heizöl schwer

Niederlande

Italien

Bundesrepublik

Kohle (DM pro $100 \mathrm{~kg}$ )
7,89

9.99

6,77

6,66

8,98

14,15

8,09
7,81

10,01

6,64

5,82

9,54

7,82

k.A.

9,51

k.A.

8,96

6,78

9,11

$\begin{array}{lrrrr}\text { Ruhr } & 14,15 & 14,45 & 14,45 & 14,64 \\ \text { USA (fot) } & 8,09 & 8,28 & 7,32 & 14,64\end{array}$

Beim volumenmäßig wichtigsten Ölprodukt, dem leichten Heizöl, liegen die USA um $25 \%$ über đem italienischen Preis, der für alle EG-Länder ohne Kohleprotektionismus repräsentativ sein dürfte. Beim schweren Heizöl steigt selbst in der Bundesrepublik der Preis keineswegs auf den Preis der Kohle, sondern liegt trotz der zusätzlichen Steuer yon 4 Pf pro 1 um mehr als $50 \%$ unter dem Preis von Ruhrkohle, wenn beachtet wird, daß erst etwa $150 \mathrm{~kg}$ Kohlen zum Preis von 21 - 22 DM den Heizwert von 100 l schwerem Heizöl haben. Außerdem liegt der Preis für schweres Heizöl auch deutlich unter dem Preis für Kohle in den USA (150 kg kosten 11-12 DM 1972. 1973).

Selbst in dem Land der alten EG, das am stärksten seine Kohle schützte, war also der Preis für schweres Heizöl niedriger als der Preis für Kohle in den USA. Angesichts des in den USA im Vergleich zur Bundesrepublik höheren Anteils der Kohle an der Primärenergieversorgung ergibt sich auch unter Einbeziehung der im Vergleich zur amerikanischen Kohle teureren Ruhrkohle gerade durch den stärkeren Rückgriff auf billiges schweres Heizöl ein niedrigerer Mischpreis auf dem Teilmarkt Kohle-schweres Heizöl in der Bundesrepublik als in den USA.

Nicht Elsenhans hat vor lauter Bäumen den Wald nicht gesehen, sondern Massarat hat die Empirie nicht erfaßt. Massarat hat aber auch nicht das Dilemma der amerikanischen Energiepolitik erfaßt. Das vor 1973 bestehende Preisniveau erlaubte nämlich nur eine sinkende Eigenversorgung. Entscheidend für die amerikanische Politik war nicht nur, ob das amerikanische Energiepreisniveau damals höher war als das der amerikanischen Konkurrenten in Westeuropa und Japan, sondern $o b$ und um wieviel es höher sein würde, falls die USA zur Förderung heimischer Energiequellen übergehen müßten (5).

4 Statisitisches Bundesamt, Reihe: Preise und Preisindizes im A usland.

$5 \quad Z u$ den Kosten alternativer Energie in den USA und Kanada, Elsenhans, $H$.: Nahostwaffe Erdöl, in: Der Bürger im Staat, Juni 1975, S. 151, Fußnote 25. 
Zugleich widerlegen diese Zahlen Massarats diesmal nur am Rand (S. 27), früher aber mit Verve vertretene These, daß die Produktionspreise der marginalen Produzenten den Marktpreis bestimmen (6) Nicht einmal auf dem Teilmarkt Kohle/schweres Heizöl steigt in der Bundesrepublik der Preis für schweres Heizöl auf den Preis der Kohle und für den Preis von leichtem Heizöl, der in allen Fällen deutlich unter dem Preis der Kohle liegt, ist eine solche Beziehung zu den marginalen Energieproduzenten nicht einmal ansatzweise feststellbar; der Grund ist, daß es durchaus andere Mittel zum Schutz der Kohle gab und gibt als die Anhebung des Preises für andere Energieträger, weil die Kohle nicht mehr der Hauptenergieträger ist.

Allgemein ist aber darauf zu verweisen, daß die von Massarat konstruierte besondere Form der Preisbildung entsprechend dem Produktionspreis des marginalen Produzenten im Bereich der Rohstoffproduk tion falsch ist. Zwar ist richtig, daß die starke Abhängigkeit des Produktionspreises von den natürlichen Bedingungen der Produktion zur Folge hat, daß sich anders als im Bereich der verarbeitenden Industrie Fortschritte in der Arbeitsproduktivität nicht automatisch über die Konkurrenz verallgemeinern. Das heißt aber nicht, daß deshalb der marginale Produzent noch einen Durchschnittsprofit erzielt. Die amerikanische Kohlenindustrie hat in den 60er Jahren permanent unterdurchschnittliche Profitraten erzielt, was den Aufkauf der Kohlegruben durch die Ölkonzerne erleichtert hat (7) Die Unmöglichkeit, einmal investiertes Kapital wieder flüssig zu machen, um es in anderen Ber eichen anzulegen, hat zur Folge, daß mit investiertem Kapital weitergearbeitet wird, auch wenn es keinen durchschnittlichen Profit erzielt; wie lange produziert wird, hängt davon ab, ob über die laufenden Kosten hinausgehende Erlöse, also überhaupt ein Profit erzielt wird. Die Bedingung, daß wenigstens der Durchschnittsprofit erzielt werden muß, gilt nur für die Neuanlage von Kapital. Entscheidend ist dann, ob der marginale Produzent zur Befriedigung der kaufkräftigen Gesamtnachfrage die Produktion steigern muß oder nicht. Diese Bedingung gilt genauso in der verarbeitenden Industrie: auch hier erzielt bei einer rasch expandierenden Nachfrage der Grenzproduzent einen Durchschnittsprofit und bei stagnierender Nachfrage nicht.

\section{Warum kam es zur Ölkrise?}

Hier wirft Massarat mir vor, die internen Entwicklungen in den ölländern nicht richtig einzuschätzen und die Position der amerikanischen Einzelkapitale und des amerikanischen Gesamtkapitals zu vermischen.

Zunächst eine Richtigstellung: Nirgends ist von mir behauptet worden, daß die Ölkonzerne von sich aus an einer Rückverlagerung der Energieproduktion in die Industrieländer interessiert waren. Deshalb habe ich ausdrücklich vor der These einer "Verschwörung der Konzerne" gewarnt. Massarat zitiert sogar auf S.33 den entscheidenden Satz, nach dem die Ölkonzerne sich erst wegen der Forderungen der Ölländer und der damit verbundenen Minderung ihrer Extraprofite bei gleichzeitiger größerer Risikoanfälligkeit der Förderung zur Rückverlagerung entschlossen hätten. Daß3 die OPEC-Länder und nicht die Konzerne als erste Ursache der Rückverlagerungsstrategie anzusehen sind, ist zwischen Massarat und mir nicht strittig, wohl aber die Einschätzung des Verhaltens des amerikanischen Staatsapparats und der Konzerne angesichts dieser Bedrohung ihrer Interessen und die Einschätzung der Ursachen für die Veränderungen in der Ölpolitik der OPEC-Länder. A uf S. 19 des von Massarat zitierten Einleitungsaufsatzes (8) heißt es: "Die Konzerne beantworteten die zunehmende politische Gefährdung mit einer Strategie (der Rückverlagerung), während die Notwendigkeit zu einer solchen Strategie aus Faktoren herrührte, die eine Kräftekonstellation auf dem Welterdölmarkt herbeifürten, aufgrund derer die Akkumulationsfonds der Konzerne (nämlich über die allmähliche Aneignung der Rente durch die Förderländer, was auf S. 17 - 18 dargestellt worden war) nicht in ausreichendem Maße wach-

6 Massarat, M.: Energiekrise oder die Krise des Kapitalismus, in: Prokla 11/12, S, 223.

7 Im Überblick Joe Stork: Middle East Oil and the Energy Crisis, in: Middle East Research and Information Project, Okt. 1973, S. 12.

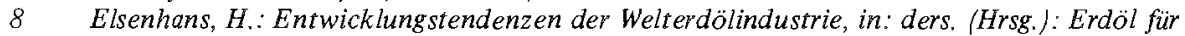
Europa, Hoffmann \& Campe. Hamburg 1974, S. 7 - 47. 
sen konnten.“ Und auf S. 23 heißt es ausdrücklich: „.. . es sicher nicht korrekt wäre, den Konzernen zu unterstellen, daß sie die Lieferbeschränkungen der arabischen Länder selbst herbeigeführt hätten . .... (9) Die auch von Klaus Busch (10) mir unterstellte Erklärung der Ölkrise als Inszenierung des amerikanischen Kapitals findet in meinen Publikationen keinen Beleg: richtig und von mir behauptet wurde, daß die Konzerne und das Gesamtkapital der USA unter der Voraussetzung, daß die Forderungen der ölländer nicht mehr abzublocken gewesen sind, für eine Strategie der Riickverlagerung optierten.

Strittig ist dann im Gegensatz zur Interpretation meiner Veröffentlichungen durch Massarat die Einschätzung der Interessenlage einzelner Kapitalfraktionen in den USA und des gesellschaftlichen Charakters der Ölexportpolitik der Förderländer. Hier wirft mir Massarat - ausgehend von seiner oben widerlegten These eines niedrigeren Energiepreisniveaus in den USA im Vergleich zu Westeuropa - vor, daß meine Analyse (es handelt sich bei mir nicht um bloße Ableitungen) der Interessenlagen der verschiedenen Kapitalfraktionen widersprüchlich sei. weil die Rückverlagerungssstrategie aus den Interessen der Ölkonzerne - Einzelkapitale - und aus dem Interesse des amerikanischen Gesamtkapitals erklärt werde, (S. 40) und ihre Heranziehung als Erklärung für ein Intresse an steigenden Ölpreisen falsch sei, weil die höheren Kosten anderer Energieträger anders und billiger hätten finanziert werden können. (S. 37 - 39)

Dem wäre zu entgegenen, daß Parallelität von Interessen zwischen Einzel- und Gesamtkapitalen kein Widerspruch ist, sondern eben erklärt, warum innerhalb des politischen Entscheidungsprozesses sich diese und nicht andere Einzelkapitale durchsetzen konnten. Wie die v. a. an billiger Energie interessierten Einzelkapitale 1969 - 1970 gegenüber den Ölkonzernen bei der Herausbildung einer Ölstrategie des amerikanischen Gesamtkapitals ausmanövriert wurden, habe ich ausführlich darge- und belegt (11).

Massarat übergeht diese Zusammenhänge und bringt als Gegenargument, daß die Verteuerung der Energiequellen im Verlauf der Rückverlagerung die Profitrate des amerikanischen Gesamtkapitals mindern müsse (S. 38). Eine solche Behauptung folgt zwar der Marxschen Verkürzung der Bestimmung der Profitrate auf die Frage: was ist verteilbar? (wobei die Frage: was ist realisierbar? ignoriert wird), ignoriert aber alle späterèn Arbeiten zur Profitratenentwicklung (12). Im Rahmen der Erwiderung auf Massarat sei nur auf den Zusammenhang zwischen Akkumulationsrate, die den Bedarf an Investitionsmitteln bestimmt, und Profitrate verwiesen (13). Wenn die Energieproduktion in den politisch sicheren Gebieten hohe Investitionsmittel langfristig etfordert, dann werden die Profitraten zumindest in der Ölindustrie steigen und dies keineswegs zu Lasten anderer Sektoren, sondern zu Lasten des privaten Verbrauchs gehen. Dabei entstehen über eine steigende organische Zusammensetzung des Kapitals, die sich auch in einem

$9 \quad$ Und noch deutlicher: „Aus der Analyse der Interessenlage der Hauptbeteiligten läßt sich allerdings nicht ableiten, daß die Konzerne die arabischen Länder angestiftet hätten, die ölkrise auszulösen. Eine solche Aussicht ist deshalb vordergründig, weil sie eine Kongrienz zwischen dem politischen Prozeß und objektiven Interessenlagen postuliert und den Me. chanismus der Adjustierung von Interessen verkennt. Viel wichtiger ist, daß sich alle Beteiligten dann, wenn jeder die Interessenlage des anderen kannte, verhalten mußten, als ob man sich geeinigt hätte. "Elsenhans, $H$.: Erdöl und Politik. Die Entwicklungstendenzen des Weltmarkts und die internationale Erdölundustrie, in: Internationale Entwicklung $1974 I I$, S. 20.

10 Busch, Klaus: Der EG Integrationsprozeß in der Krise, aus: Links Sozialist ische Zeitung, Mai 1975, S. 15; auch in: Voll, Klaus: Weltmarkt und Peripherie. PH Berlin 1974, S. 59.

11 Elsenhans, H.: Entwicklungstendenzen, a.a.O., S. 22.

12 VgL z. B. Robinson, Joan: Der tendenzielle Fall der Profitrate, in: Rolshausen, C. (Hrsg.): Eine Kontroverse um das Gesetz des tendenziellen Falls der Profitrate. EVA. Frankfurt 1970, S. 41 f. Wood, Adrian: A Theory of Profits. Cambridge University Press. Cambridge 1975, v.a. S. 108. Kregel, J. A.: Rate of Profit, Distribution and Growth: Two Views. Mac Millan, London 1971, v.a. S. 145.

$13 \mathrm{Vgl}$. dazu Elsenhans, H.: Reallohnsteigeningen - Chance des Kapitalismus? Zum Gesetz des tendenziellen Falls der Profitrate, in: Forum DS, Nr. 2 
steigenden Kapitalkoeffizienten niederschlägt, günstige Akkumulationsbedingungen, die Wirtschaftswachstum ohne proportionale Lohnsteigerungen erlauben, wie dies Lenin in flagrantem Gegensatz zur Marxschen Profitratentheorie in der "Entwicklung des Kapitalismus in Rußland“ gezeigt hat (14). Das amerikanische Gesamtkapital muß also keineswegs sinkende Profitraten aus der steigenden organischen Zusammensetzung des Kapitals befürchten, sondern kann sehr wohl mit neuen rentablen Anlagemöglichkeiten rechnen. Um aber neuen Mißverständnissen vorzubeugen: Ich behaupte nicht, daß das amerikanische Gesamtkapital deshalb für die Rückverlagerungssstrategie eingetreten sei: Der ideelle Gesamtkapitalist ist eine Fiktion - realiter entsteht er als Integration divergierender Interessen, die auf der Grundlage der Einschätzung des Wünschbaren und des Machbaren definiert werden und in die deshalb die Erwartungen über das Verhalten der anderen Akteure (z. B. der OPEC-Länder) eingehen (15). Und als Integration von Interessen gehen in die Definition der Interessen des Gesamtkapitals sehr verschiedene Erwartungshorizonte ein, so daß der Rationalitätsgrad dieser Interessendefinition, d. h. der Grad, zu dem die Interessendefinition langfristigen Perspektiven zugeordnet wird, unterschiedlich ist.

Nicht die Erhöhung der organischen Zusammensetzung des Kapitals in der amerikanischen Wirtschaft, sondern die im Vergleich zu den Konkurrenzen in Europa und Japan höheren Energiek osten konnten die Profitraten sowohl der rückverlagernden Energieproduzenten als auch der amerikanischen energieverbrauchenden Unternehmen senken. Wenn für alle die Energie teurer wurde, stiegen die Produktionskosten für alle, so daß die höheren Produktionskosten je nach Preis- und Einkommenselastizität der Nachfrage abgewälzt werden konnten. Wenn die Energie nur für amerikanische Produzenten teurer wurde, dann bestand die Gefahr der Verbesserung der Konkurrenzfähigkeit von in Westeuropa und Japan mit billiger Energie produzierenden Unternehmen. Falsch ist, daß dies über eine Subventionierung im Rahmen der US-Wirtschaft hätte leichter aufgefangen werden können als über eine Verteuerung des OPEC-Öls. Im Fall von Subventionen hätten andere Teile der US-Industrie bzw. die Lohnabhängigen in den USA die zusätzlichen Mittel aufbringen müssen, nicht jedoch die Unternehmen in Japan und in der EG. Tatsächlich wurde vor der Ôlkrise erwogen, die westeuropäischen Regierungen zur Subventionierung der Erschließung alternativer Energiequellen in den USA zu bewegen (16). Die nach der Ölkrise einsetzende Auseinandersetzung über den Mindesteinfuhrpreis in die Länder der internationalen Energiebehörde (IEA) belegt die Fortdauer des Interesses des amerikanischen Gesamtkapitals an einem dauerhaft hohen Energiepreis für europäische und japanische Produzenten. Daß vor Oktober 1973 die amerikansichen Ölpolitik allerdings an einer langsameren und planvolleren Steigerung des Ölpreises interessiert war, ist einzuräumen.

Wenn von mir nicht behauptet wurde, daß die Ölkonzerne bzw. die US-Regierung von sich aus ein Interesse an steigenden Ölpreisen entwickelt haben, so muß gefragt werden, warum die Öländer einen Druck in Richtung auf höhere Preise für Öl ausübten.

Hier wird Massarats Argumentation grotesk (S. 29 ff). Weil sich kapitalist ische Produktionsverhältnisse in den repräsentativen OPEC-Ländern (S. 31) durchgesetzt hätten, würden sich die herrschenden Gesamtkapitale in diesen Ländern nicht mehr wie vorkapitalistische Klassen mit einem Tribut begnigen (S. 29), sonden verlangten die Differentialrente. Bei der Frage, wie diese angeeignet werden könne, erfahren wir die Bedingung, das in der überwiegenden Zahl der Förderländer sich kapitalistische Produktionsverhältnisse entwickelt hätten (S.31) und daß3 die Existenz eines Kartells die Durchsetzung der Forderungen erlaubt.

Zunächst ist festzuhalten, daß der $\mathbb{K}$ ampf um die Rente die monopolistìsche Verknappung des Angebots an billiger Energie erfordert, Rente immer ein Element des Monopols enthält (auch die Marxsche Rente, die absolute Grundrente ein gesellschaftliches Monopol, die Diffe-

\footnotetext{
14 Lenin, Werke 3, S. 44.

15 Diese Überlegungen zum Interessenbegriff habe ich ausgefürt in Elsenhons, H.: Frankreichs Algerienkrieg 1954-1962. Entkolonisierungsversuch einer kapitalistischen Metropole. Honser. Müchen 1974, $9.159 \mathrm{f}$.

16 Lévi, Mario: Colloque franco-allemand sur l'énergie. Rouen 4-5 mai 1973, in: Politique étrangere, 3/1973, S. 355.
} 
rentialrente ein ,natürliches" Monopol) (17). Die im Vergleich zum technisch möglichen Angebot an billigem öl geringe Weltnachfrage erlaubte - dies zeigen die Preissenkungen für öl auf dem japanischen und dem westeuropäischen Markt in den 60er Jahren - eine Erhöhung der Ölpreise auf den Kostpreis teurer Energie aus den OECD-Ländern nur, wenn alle Anbieter billiger Energie sich zusammenschlossen. Der dadurch mögliche Einsatz von außerökonomischem $Z$ wang in der Form Exportmengenbegrenzung wurde von den Konzernen in ihrer Rückverlagerungsstrategie antizipiert (die gerade verhindern sollte, daß über das Monopol der Preis noch über die mittel- und langfristigen Kosten der Energieproduktion in den kapitalistischen Industriekländern steigen konnte). Die Durchsetzung außerökonomischen Zwangs erfordert allerdings politisch Handlungsspielräume (um z. B. die Anwendung außerökonomischen Zwangs im Gegensatz seitens der kapitalistischen Industrieländer zu verhindern), deren Berücksichtigung Massarat meiner Analyse vorwirft. Er müßte aber dann die Frage beantworten, warum anders als 1956 und 1951 - 54 die kapitalistischen Industrieländer nicht militärisch intervenierten, um die Anwendung außerökonomischen. Zwangs seitens der Förderländer zu verhindern. Sobald er dies versucht, wird er meine Analyse des internationalen Kräfteverhältnisses unter besonderer Berücksichtigung des Nahen Ostens übernehmen müssen.

Bleibt die Frage, warum die Ölländer zu außerökonomischem Zwang übergehen: Sicher nicht, weil sie kapitalistisch geworden sind. Die Erhebung von Monopol- und Differentialrenten war ein Kennzeichen des Handels der vorkapitalistischen Klassen in den Gesellschaften Asiens und Afrikas vor deren ,,Öffnung" für den europäi schen Handel. Die Kriege ab 1815 (Öffnung der Türkei, Opiumkrieg) dienen bekanntlich der Durchsetzung des Freihandels und damit der Beseitigung von Monopolrenten. Genauso haben in der Zwischenkriegszeit die politisch selbständigen Ölländer wie der Iran Abgabenerhöhungen durchzusetzen versucht und dazu sogar den Völkerbund eingeschaltet, obwohl z. B. dieses Land nach Massarat damals noch nicht als kapitalistiscli anzusehen ist. Die Forderung nach Aneignung von Monopolrenten bzw. Differentialrenten ist also historisch keineswegs mit dem Aufstieg einer kapitalistischen Klasse verbunden.

Bei genauerer Lektüre der Massaratschen Texte zeigt sich dann, daß er. als Kapitalismus in den Ölländern die Fähigkeit der in diesen Ländern herrschenden Klassen ansieht, rechnen zu können. „,Die herrschenden Klassen der ölproduzierenden Staaten hören auf, die Öleinnahmen als bloßes Revenue zu betrachten ... Das Bewußtsein wächst in dem Maße, wie sie einen Einblick in die Zusammenhänge der Kapitalverwertung erhalten" (S. 29, Hervorhebungen H.E.). Während Kapitalisten sonst in der Theorie des Kapitalismus akkumulieren, weil sie sonst durch die Konkurrenz ihr Kapital verlieren, akkumulieren sie in der Massaratschen These, weil sie rechnen gelernt haben, also aufgrund eines nicht über die Reproduktions- und Verwertungsbedingungen des Kapitals bestimmten soziokulturellen Wandels. Diese neue Form von Modernisieru ngstheorie verwundert bei einem Autor, der sich permanent auf seine ökonomischen Ableitungen beruft.

17 Hier wäre Massarat einzuräumen, daß die von ihm (S. 50 f.) kritisierte Ablehnung des Begriffs der absoluten Grundrente verkürzt formuliert worden ist: Richtig ist aber, daß die Höhe der Differentialrente von den Kosten des Produzenten mit den ungünstigsten natürlichen Produktionsbedingungen abhängt, sofern er diese Kosten bezahlt. Massarats Argument, daß die kapitalistischen Industrieländer durch eine Subventionierung der teuren Energiequellen das Ansteigen des Ölpreises hätten verhindern können, wird sinnlos, wenn nicht angenommen wird, daß dadurch die Kosten der Produzenten mit den ungünstigsten natürlichen Bedingungen gesenkt werden. Die , absolute" Grundrente ist im übrigen Ausdruck der unvollständigen Durchsetzung der Konkurrenz. Amüsanterweise druckt die Prokla anschließend an Massarats Artikel in der gleichen Nummer die Arbeit von Birgit Schultz: Ökonomische Voraussetzungen bäuerlicher Interessenvertretung ab, in dem diese These von Amin/Vergepoulos (Amin, Samir/Vergepozlos, Kostas: La question paysanne et le capitalisme. Anthropos. Paris 1974, S. 183 -187) übernimmt, nach der der Kleinbauer und potentiell auch mittlere Bauern weder einen Durchschnittsprofit noch eine Bodenrente erzielen. 
Demgegenüber argumentiere ich allerdings nicht nur mit dem Hinweis auf die Interessen der Unterprivilegierten (19). Ich gehe davon aus, daß die peripheren Kapitalismen aufgrund der ungleichen Einkommensverteilung und der mit ihr wechselseitig bedingten Abhängigkeit nur begrenzt akkumulationsfähig sind. Aus diesem Grund erweitert sich laufend der staatliche Sektor, der die Reproduktions- und Akkumulationskrise überwinden soll. Dieser Sektor reagiert auf die soziale Krise und den dadurch ausgelösten Druck von unten mit Teilreformen und Repression, je nach verfügbaren Mitteln, und versucht die Unterprivilegierung der Massen, soweit sie nicht mehr ignoriert werden kann, durch Zufuhr äußerer Ressourcen und nationalistische Einstellungen gegenüber dem zum Sündenbock deklarierten ausländischen Kapital zu kanalisieren. In diesem Zusammenhang sind Tendenzen zur Herausbildung von Gesellschaftsformationen zu beobachten, die den APW's ähneln. Eine Staatsklasse kontrolliert einen erheblichen Teil des gesellschaftlichen Mehrprodukts, verpraßt dieses oder investiert dieses (allerdings nicht gebunden an kurzfristige Rentabilitätserwartungen), je nach dem Grad der Bindung an die Interessen der Unterprivilegierten, wie dies auch in den APW's - gute und schlechte - Kaiser - der Fall war. Diese Analyse deckt Fälle wie Algerien (Beispiel einer starken Bindung der Staatsklasse an die Interessen der Unterprivilegierten) und Iran (Beispiel einer schwachen Bindung der Staatsklasse an die Interessen der Unterprivilegierten).

Daß solche Gesellschaftsformationen die Probleme der Verfügbarkeit von Mehrprodukt für Luxuskonsum oder Investitionen über die Anwendung außerökonomischen Zwangs zur Aneignung von Renten lösen können, ist angesichts der Kontrolle des Mehrprodukts und des AuBenwirtschaftsverkehrs durch eine (in Unterschied zu einer Bourgeoisie nicht in individuelle Kapitalbesitzer, die miteinander in Konkurrenz stehen) nicht fraktionierte Staatsklasse leicht verständlich. Es ist keineswegs der zunehmende kapitalistische Charakter der Produktionsweise in den Ölländern, sondern die über das Eindringen kapitalistischer Sektoren ausgelöste Akkumulations- und Reproduktionskrise (Entwicklung von Unterentwicklung, Immisering Growth), die zu kompensierenden Maßnahmen und dabei zur Herausbildung eines Staatsapparats mit der Möglichkeit der Bildung einer Staatsklasse führt, die die Grundlage für den erhöhten Druck der Ölländer in der Preisfrage bildet. Die Parallelität dieser Entwicklung sowohl in den Gesellschaften, in denen ,traditionelle" Eliten diese Funktionen besetzen, als auch dort, wo national- und selbst sozialrevolutionäre Befreiungsbewegungen die Macht übernommenhaben, bildet die Grundlage für die Zusammenarbeit von Regimen sehr unterschiedlicher innenpolitischer Zielsetzung und Praxis.

\section{Politologischer Reformismus?}

Wenn in den inhaltlichen Aussagen Massarats Kritik sich als gegenstandslos erweist, dann ist zu fragen, inweiweit die theoretische Basis, auf die er sich beruft, tragfähig ist. Massarat definiert sein Herangehen als politökonomisch, gegenüber der politologischen Analyse, die er mir unterstellt (S. 49). Dieser politökonomische Ansatz führt - abgesehen von der fehlerhaften Empirie in der Frage des Energiepreisniveaus, zu Aussagen wie

- ich hätte den kapitalistischen Charakter der OPEC-Strategie nicht erkannt,

- ich hätte das Verhältnis zwischen Einzelkapitalen und Gesamtkapital nicht bestimmen können.

Im ersten Fall handelt es sich bei Massarats Analyse um den Versuch, das sogenannte FünfStadien-Schmea (Urgemeinschaft - Sklaverei - Feudalismus - Kapitalismus - Sozialismus) auf die Länder der Dritten Welt zu übertragen. Die reale Entwicklung, nämlich die Tendenzen zur Rekonstituierung von APW's neuen Typs, die ich versuchsweise unter dem vielleicht unglückli-

19 Diesen Argumentationsgang habe ich ausgeführt: in Elsenhans, H.: Zur Rolle der Staatsklasse bei der Überwindung von Unterentwicklung, in: Schmidt, Alfred (Hrsg.): Strate. gien gegen Unterentwicklung. Campus, Frankfurt 1976, S. 250-265; und Elsenhans, H.: Die Statsbourgeoisie/Staatsklasse in den unterentwickelten Ländern zwischen Privilegierung und Legitimationszwang. Kongreß der DVPW. Duisburg Okt. 1975. 
chen Begriff der ,staatsabhängigen Entwicklungsgesellschaft" (20) gefaßt habe, ignoriert er. Tatsächlich wechselt er ohne jede Vermittlung von der Theorie der Grundrente - die zwischen uns nicht strittig ist - auf die Theorie der Stadien der gesellschañtlichen Entwicklung, die er der Theorie der Rente nur aufklatschen kann, indem er den Kapitalismus darauf reduziert, daß die herrschende Klasse rechnen kann (S. 29).

Der methodologisch kreative Ansatz von Marx des Zusammenhaltens von Totalität in Struktur, Genese und Entwicklungstendenzen wird aufgegeben, indem die politische Vermittlung von ökonomischer Dynamik in Gesellschaftsstrukturen und politisches Handeln innerhalb gesellschaftlich bedingter Kräfteverhältnisse reduziert wird auf ein Nebeneinander zwischen ökonomischer (im einzelnen nicht empirisch abgesicherter) Analyse und dogmatischer Gesellschaftstheorie (dogmatisch, weil sich nicht der Realität vermittelnd und aus der Analyse der Realität wieder zu begrifflichen Abstraktionen aufsteigend). Die postulierte Notwendigkeit der ,Einbeziehung der politischen Ebene" (S. 25) wirkt dann bloß deklamatorisch. Die Folge ist dann ein bloß theoretisierender Praxisbezug. Sicher hat Massarat zumindest in meinem Fall recht, wenn er mich unter die Reformisten innerhalb des Spektrums der Linken in der Bundesrepublik einreiht (obwohl mir unklar ist, ob als Reformist der Autor noch Teil der Linken ist, zumindest jener in Kapellen und Sekten aufgespaltenen Linken, die andere immer in Schubladen steckt). Wie eine Umstrukturierung der Gesellschaften der Peripherie aussehen muß, habe ich an anderer Stelle gezeigt, nämlich Umstrukturierung des Produktionsapparats, damit Massen für Massenbedürfnisse produzieren (21). Daß dabei auch Kategorien der Rechenhaftigkeit - Gewinn, Kostenkontrolle etc. - eine wichtige Rolle spielen, ist unbestreitbar, weil die Kategorie der gesellschaftlich notwendigen Arbeit in jeder auf Gleichheit - und dann besonders in einer auf nicht nur formalrechtliche Gleichheit angelegten Gesellschaft von zentraler Bedeutung ist (22). Indem Massarat die Rechenhaftigkeit zum Definiens des Kapitalismus macht und dazuhin den Preis (Produktionskosten + Differentialrente) zum Wert erhebt (23), darf gefragt werden, ob die mir vorgeworfene Kapitalverhaftetheit aus der Ablehnung der Kategorie der gesellschaftlich notwendigen Arbeit auch für postkapitalistische Gesellschaften seitens Massarat resultiert.

Massarat versucht den Vorwurf der Kapitalverhaftetheit weiterhin mit dem Argument zu stützen, daß er die geforderte Kooperation zwischen den Förderländern und den westeuropäischen Ländern als Versuch interpretiert, die Ölländer in kapitalistische Abhängigkeit von Westeuropa zu bringen (S. 53). Wiederholt habe ich in Arbeiten den Standpunkt vertreten, daß diese Kooperation sich von westeuropäischer Seite aus v. a. an die sozialrevolutionären und sozialreformerischen Regime richten muß, weil nur diese in der Lage sind, die Renteneink ommen im Interesse der breiten Massen in den Förderländern ausgeben zu können (24). Von der Forderung

20 Elsenhans, H.: Algerien, in: Nohlen, Dieter/Nuscheler, Franz: Handbuch der Dritten Welt. $\dot{B}$ d. 1. Hoffmann \& Campe, Hamburg 1975, S. $162-189$.

21 Elsenhans, H.: Die Überwindung von Unterentwicklung, in: Nohlen, Dieter/Nuscheler, Franz: Handbuch der Dritten Welt. Bd. 1. Hoffmann \& Campe, Hamburg 1975, S. 162 189.

22 Vgl. z. B. meine Kritik des Staatsbetriebes in Elsenhans, H.: Algerien, a.a.O., S. 76.

23 Das gilt für Massarat, M.: Energiekrise, a.a.O., S. 244, und für Massarat, M.: Linksbürgerliche Analysen, a.a.O., S. 27: „Der Wert, richtiger der Marktwert, und daher der Marktpreis..." Die Differentialrente wird damit Teil des Werts wie in der subjektiven Werttheorie und damit im Gegensatz zur Marxschen Arbeitswerttheorie. Der Unterschied ist von großer politischer Bedeutung: Ist die Aneignung der Differentialrente im ölexport ein Transfer von Wert aus den Importländern auf die Exportländer, gibt es hier die Grundlage für eine internationalistische Kritik seitens der Arbeiterklassen in den Industrieländern in der Frage der Verwendung der Gelder; wenn die Differentialrente ein Teil des Werts des Öls ist, dann ist eine solche Kritik politisch weniger zu rechtfertigen.

24 Auf den Autor bezieht sich folgendes Zitat aus dem Bericht Mario Lévis, a.a.O., S. 356, das in der gewählten Terminologie nichtganz der vertretenen These entspricht: ,Peut-etre, a fait remarquer un participant, la situation serait differente, si des gouvernements ayant des conceptions révolutionnaires et modernisatrices prenaient la place des gourvernements 
nach verstärkter kapitalistischer Durchdringung der ölländer sind meine Thesen also weit entfernt. Allenfalls kann Massarat behaupten, daß durch diese Strategie auch sozialrevolutionäre und sozialreformerische Regime (die Massarat, wie er am Beispiel seiner Einschätzung Algeriens zeigt, als Formen kapitalistischer Entwicklung ansieht, S. 31) wieder in den Kreis der kapitalistischen Länder eingegliedert werden. Hier wäre allerdings einzuwenden, daß, weil die Aneignung von Differentialrenten nicht Aneignung von im Förderland produzierten Werts ist, sondern eine Übertragung von Arbeitswert aus dem O̊̉l kaufenden Land ist (wobei noch zu klären wäre, inwieweit die Ableitung von Werten auf der Grundlage des empirisch festgestellten Werts der Arbeitskraft im internationalen Handel ohne eine Kritik des unterschiedlichen Reallohnniveaus möglich sind), dann kritisiert Massarat mit der Ablehnung der Forderung nach einer Kooperation zwischen Westeuropa und den Förderländern die Möglichkeit für die Förderländer, sich aus externen Quellen einen Akkumulationsfonds zur beschleunigten Entwicklung von Produktivkräften zu verschaffen. Massarats prinzipielle Ablehnung des Handels zum gegenseitigen Vorteil (S. 53) ist immerhin ein origineller Beitrag zur sozialistischen Theorie, erst recht, wenn dabei abgelehnt wird, diesen Handel zugunsten der progressiven Regime in der Dritten Welt zu beeinflussen. Daß gerade auch von Ländern, denen Massarat das Adjektiv sozialistisch nicht verweigern durfte (Vietnam, China, UdSSR) (25), diese externe Akkumulationsquelle des Rohstoffexports genutzt wird, und zwar mit besonderer Stützung auf die mittleren unter den kapitalistischen ländern, sollte zum Nachdenken veranlassen. Und wenn diese Kooperation zum Transfer von Ressourcen in die weniger entwickelten Länder im Interesse größerer Gleichheit notwendig ist, dann liegt ein Eintreten für diese Kooperation primär in điesem Interesse, auch wenn dabei gleichzeitig Interessen bestimmter Kapitalfraktionen berücksichtigt werden. Daß andererseits dieser Transfer von Ressourcen überhaupt nicht zur Überwindung von Unterentwicklung führen muß, ja sogar wegen des größeren verfügbaren Gesamtprodukts den privilegierten Klassen erlaubt, sich wirkungsvoller Tendenzen zu gesellschaftlichem Strukturwandel zu widersetzen, ist von mir nie bestritten worden. Gerade deshalb trete ich dafür ein, daß sowohl bei der Rohstoffrage wie beim Zugang zu den Märkten in den Industrieländern die Linke in den kapitalistischen Industrieländern fordert, daß hohe Rohstoffpreise und Absatzmöglichkeiten primär jenen Ländern zugute kommen, die aufgrund der Verwendung der Erlöse für eine Strategie der Überwindung von Unterentwicklung, die gesellschaftliche Strukturreformen einschließt, verwenden. Dabei kann sich die Linke auf das Argument stützen, daß nur im Fall der Verwendung der Exporterlöse im Interesse der Massen die Rohstofflieferungen aus der Dritten Welt auch langfristig politisch sicher sind.

conservateurs achuls, dès lors que de tels gouvernements seraient plus enclins d poursuivre des programmes d'investissements pour assure le developpement des économies nationgles". Ich habe also sehr wohl schon vor der ölkrise die Forderung vertreten, daß, weil die Steigerung des Ölpreises unausweichlich ist, die kapitalistischen Länder Westeuropas ein Interesse an der Kooperation mit revolutionären Regimen im arabischen Raum hätten. Vgl. auch Elsenhans, H.: Sicherung des Energiebedarfs in Westeuropa nur durch progressive panarabische Kräfte, in: epd-Entwicklungsdienst, Mai 1974, S. 5 - 17.

25 Zur UdSSR: Sutton, Anthony C.: Western Technology and the Soviet Economic Development. 1917 to 1930. Hoover Institution Publications. Stanford (California) 1968, S. $44 \mathrm{ff}$. Zur Gewährung von Ölkonzessionen durch Vietnam, The Petroleum Economist, Juni 1976, S. 234, und Sept. 1975, S. 339. Zu China, ebenda, Okt. 1975, S. 391, Le Monde, 24.10.1976, S. 40. 